\title{
Improved Geometry of Decellularized Tissue Engineered Heart Valves to Prevent Leaflet Retraction
}

\author{
Bart Sanders, ${ }^{1,2}$ Sandra Loerakker, ${ }^{1,2}$ Emanuela S. Fioretta, ${ }^{1}$ Dave J.P. Bax, ${ }^{3}$ \\ Anita Driessen-Mol, ${ }^{1,2}$ Simon P. Hoerstrup,${ }^{1,4}$ and Frank P. T. Baaijens ${ }^{1,2}$ \\ ${ }^{1}$ Department of Biomedical Engineering, Eindhoven University of Technology, Postbus 513, 5600 MB Eindhoven, The \\ Netherlands; ${ }^{2}$ Institute for Complex Molecular Systems, Eindhoven University of Technology, Eindhoven, The Netherlands; \\ ${ }^{3}$ Equipment \& Prototype Center, Eindhoven University of Technology, Eindhoven, The Netherlands; and ${ }^{4}$ Swiss Center for \\ Regenerative Medicine, University Hospital of Zürich, Zurich, Switzerland
}

(Received 2 May 2015; accepted 7 July 2015; published online 17 July 2015)

Associate Editor Jane Grande-Allen oversaw the review of this article.

\begin{abstract}
Recent studies on decellularized tissue engineered heart valves (DTEHVs) showed rapid host cell repopulation and increased valvular insufficiency developing over time, associated with leaflet shortening. A possible explanation for this result was found using computational simulations, which revealed radial leaflet compression in the original valvular geometry when subjected to physiological pressure conditions. Therefore, an improved geometry was suggested to enable radial leaflet extension to counteract for host cell mediated retraction. In this study, we propose a solution to impose this new geometry by using a constraining bioreactor insert during culture. Human cell based DTEHVs $(n=5)$ were produced as such, resulting in an enlarged coaptation area and profound belly curvature. Extracellular matrix was homogeneously distributed, with circumferential collagen alignment in the coaptation region and global tissue anisotropy. Based on in vitro functionality experiments, these DTEHVs showed competent hydrodynamic functionality under physiological pulmonary conditions and were fatigue resistant, with stable functionality up to 16 weeks in vivo simulation. Based on implemented mechanical data, our computational models revealed a considerable decrease in radial tissue compression with the obtained geometrical adjustments. Therefore, these improved DTEHV are expected to be less prone to host cell mediated leaflet retraction and will remain competent after implantation.
\end{abstract}

Keywords-Heart valve replacement, Tissue engineering, Computational modeling, Decellularization, Bioreactor.

\section{INTRODUCTION}

Annually, approximately 280.000 patients worldwide undergo either a mechanical- or bio-prosthetic heart valve transplantation. ${ }^{20}$ Although these are life saving devices, the lack of growth potential of these prostheses is a major problem for pediatric patients. They have to go through multiple staged interventions to accommodate the increased annulus size, with increasing morbidity and mortality risks. ${ }^{22}$ Therefore, there is an urgent need for heart valve prostheses with growth capacity that last a lifetime. ${ }^{3,21}$

Decellularized tissue-engineered heart valves (DTEHVs) might represent a promising alternative. From our first long-term in vivo experiments, where we implanted DTEHVs in sheep and non-human primates, we learned that the DTEHVs start to repopulate after $5 \mathrm{~h}$, accompanied by changes in the extracellular matrix after 8 weeks of implantation. Moreover, there was ECM production over time, indicative for tissue regeneration and growth potential. ${ }^{6,30}$ This in contrast to decellularized xenogeneic heart valves, which only show limited host cell repopulation. ${ }^{7,13}$ Besides, these DTEHVs could be available off-the-shelf. ${ }^{5}$ Although these results are promising, there were signs of leaflet shortening and fusion of the leaflets with the wall, ultimately resulting in valvular insufficiency, an effect which is also reported by other groups. ${ }^{8,10}$ An explanation for this leaflet-fusing and shortening problem might be found in the valve geometry. It was shown from computational simulations by Loerakker et al. ${ }^{15}$ that the leaflets in this original valve design were subjected to tissue compression in radial direction when loaded under 
physiological pulmonary pressures. It might explain why this particular valve geometry, in combination with infiltrated host cell induced remodeling, eventually resulted into reduced leaflet size. Based on these findings, Loerakker et al. also suggested an improved valve geometry that should enable radial leaflet extension during hemodynamic loading to counteract for cellular retraction forces. This required a more profound belly curvature, enhanced coaptation area and predominantly circumferential collagen orientation. ${ }^{15}$ However, controlling the geometry of tissue engineered heart valves (TEHVs) during culture was limited thus far. Regardless of the initial shape of the scaffold starter matrix, tissue compaction occurred in all possible unconstrained directions in response to the traction forces exerted by the vascular derived cells (myofibroblasts) used to culture the valves. ${ }^{29}$ This resulted in a flattened leaflet configuration, and absence of coaptation area after culture. ${ }^{12,17}$

Therefore, the aim of this study is to find a solution to be able to improve, impose and maintain the DTEHV geometry, in accordance with the suggested geometry from the computational simulations, to reduce leaflet tissue compression in radial direction under pulmonary loading conditions. A bioreactor insert matching the improved geometry was developed, which will function as an overall geometric constraint during culture. In this way, the leaflets will compact themselves around the bioreactor insert, and when removing the insert after the decellularization procedure, the DTEHV is likely to maintain its shape. This makes it possible to design, impose and maintain the desired DTEHV geometry. Human cell-based DTEHVs were produced, and their functionality and stability were assessed using hydrodynamic and fatigue in vitro tests. The effects of the bioreactor insert on tissue formation and collagen orientation were investigated using histology and confocal microscopy. Furthermore, the mechanical properties were analyzed to investigate the degree of tissue anisotropy and used as input for computational simulations on leaflet tissue loading behavior, in order to analyze the radial strain distribution in the newly designed DTEHV.

\section{MATERIALS AND METHODS}

\section{Insert Manufacturing and Positioning}

Based on the mathematical description of Hamid et $a{ }^{11}{ }^{11}$ the original valve design was improved by adding coaptation and increasing the curvature in the belly region as being previously described by Loerakker et $a l .{ }^{15}$ This improved geometry was exported as a STEP file from the simulation software (Abaqus 6.10
Simulia, USA) and imported into computer-aided design software (Autodesk Inventor, USA), to make a one-piece component of $27.8 \mathrm{~mm}$ in length and $29.7 \mathrm{~mm}$ in diameter, which was compatible with the diastolic pulse duplicator (DPD) bioreactor system. ${ }^{17}$ The bioreactor inserts were made out of a solid piece of polyether ether ketone (PEEK) by using computer controlled milling technology.

The insert was positioned at the arterial side of the valve to enable tissue compaction around the individual posts. Small holes $(0.5 \mathrm{~mm}$ in diameter and $1 \mathrm{~mm}$ spacing) are covering the insert wall to facilitate nutrient exchange with the adjacent tissue. Three large triangular shaped openings on top were incorporated for medium exchange towards the tissue-engineered valvular wall.

\section{Heart Valve Tissue Engineering}

Tissue-engineered heart valves (TEHVs) $(n=5)$ were cultured as previously described. ${ }^{17}$ In short, trileaflet heart valves were cut from non-woven polyglycolic-acid meshes (PGA; thickness $1.0 \mathrm{~mm}$; specific gravity $70 \mathrm{mg} / \mathrm{cm}^{3}$; Cellon, Luxembourg), sewn (Prolene 6-0, Ethicon, USA) into a radially self expandable nitinol stent (length $=31 \mathrm{~mm}$, ID $=30 \mathrm{~mm}$ at $37^{\circ} \mathrm{C}$; PFM-AG, Germany), and coated with $1 \%$ poly-4hydroxybutyrate $\left(\mathrm{P} 4 \mathrm{HB}\right.$; MW: $1 \times 10^{6}$; TEPHA Inc., USA) in tetrahydrofuran (THF; Sigma-Aldrich, USA). The heart valve shaped constructs had an initial coaptation length of $5 \mathrm{~mm}$ and a maximal radial belly length of $19 \mathrm{~mm}$. These constructs were sterilized with $70 \%$ ethanol (EtOH, VWR international S.A.S. Fontenay-Sous-Bois, France) for $15 \mathrm{~min}$, washed 3 times with phosphate buffered saline (PBS) for $10 \mathrm{~min}$, incubated in an antibacterial-anti fungus solution $10 \%$ penicillin/streptomycin (Pen/Strep) (Lonza, Belgium), with $0.5 \%$ fungin (InvivoGen, USA), for $30 \mathrm{~min}$, and washed 3 times with PBS for $10 \mathrm{~min}$. Hereafter, the valves were incubated overnight in growth medium (Advanced Dulbecco's Modified Eagle Medium, Gibco, USA), supplemented with $10 \%$ fetal bovine serum (FBS, Biochrom, Germany), 1\% Pen/Strep and 1\% Glutamax (Gibco, USA). Primary isolated human vascular-derived cells were harvested from the human vena saphena magna from a 77-year-old patient, according to the Dutch guidelines for secondary used materials, and seeded $\left(0.3 \times 10^{6}\right.$ cells $/ \mathrm{cm}^{2}$, passage 6$)$ into the valvular shaped scaffolds using fibrin as a cell carrier. The seeded constructs were placed into the DPD bioreactor system together with the newly developed insert, containing growth medium supplemented with L-ascorbic acid 2-phosphate $(0.25 \mathrm{mg} / \mathrm{mL}$, Sigma, USA). Pulsatile flow was applied at $1 \mathrm{~Hz}$ to the unshielded ventricular side of the valve. 


\section{Decellularization Procedure}

After 4 weeks of culture, the obtained TEHVs $(n=5)$ were decellularized as described by Dijkman et al. ${ }^{5}$ Briefly, TEHVs were washed 3 times $10 \mathrm{~min}$ with PBS and decellularized overnight in detergent solution $(0.25 \%$ Triton X-100, sodium deoxycholate and $0.02 \%$ EDTA), where after the bioreactor insert was removed. Nucleic remnants were enzymatically degraded by using Benzonase (EMD Millipore, USA) incubation steps, diluted in $50 \mathrm{mM}$ TRIS-HCL buffer solution in concentrations of 100,80 and $20 \mathrm{U} / \mathrm{mL}$ for 8,16 and $8 \mathrm{~h}$, respectively, on a shaker at $37^{\circ} \mathrm{C}$. Afterwards, the DTEHVs were washed 3 times with PBS and incubated for $24 \mathrm{~h}$ in M-199 medium (Gibco, USA) on a shaker at $4{ }^{\circ} \mathrm{C}$ to remove cellular remnants. Valves were washed 3 times with PBS, sterilized with $70 \% \mathrm{EtOH}$ for $15 \mathrm{~min}$, washed 3 times with PBS, and incubated for $30 \mathrm{~min}$ with an anti-fungi/bacterial solution. After sterilization, the DTEHVs were stored at $4{ }^{\circ} \mathrm{C}$ until further use.

\section{In-Vitro Valve Functionality}

Out of the 5 DTEHVs, 4 were used for in vitro valve functionality assessments, and the remaining valve served as a control, not subjected to fatigue testing.

\section{Hydrodynamic Pulsatile Functionality Assessment}

DTEHVs $(n=4)$ were placed inside a silicon annulus of $30 \mathrm{~mm}$ inner diameter and positioned into a hydrodynamic pulsatile test system (HDT-500, BDC Laboratories, USA) containing a physiologic saline solution at $37{ }^{\circ} \mathrm{C}$. Valves were subjected to physiological pulmonary conditions (rate of $72 \mathrm{BPM}$, stroke of $70 \mathrm{~mL}$, maximum diastolic pressure difference of $25 \mathrm{mmHg}$ ) for $1 \mathrm{~h}$. Flow and pressures were measured using a transonic sensor (TS410, Transonic Systems, USA) and pressure sensors (BDC-PT, BDC Laboratories, USA), respectively. Data was collected for $3 \mathrm{~s}$ at $5 \mathrm{kHz}$, and functionality was assessed from an average over 10 cardiac cycles by using Statys ${ }^{\mathrm{TM}}$ software (BDC Laboratories, USA), to determine the regurgitation fraction, the effective orifice area (EOA), and cardiac output (CO). The regurgitation fraction was defined as being the sum of the leakage volume and the closing volume, expressed as a percentage of the stroke volume. Slow-motion movies were recorded at high-speed burst mode to assess opening and closing behavior of the valves in motion (G15 PowerShot, Canon, USA).

\section{Fatigue Assessment}

DTEHVs $(n=4)$ were placed inside a $30 \mathrm{~mm}$ diameter silicon annulus and positioned into a valve durability tester (VDT-3600i, BDC Laboratories,
USA) containing physiologic saline solution at $37^{\circ} \mathrm{C}$. Valves were subjected to accelerated opening and closing cycles at a frequency of $10 \mathrm{~Hz}$ and a stroke between 1.20 and $2.10 \mathrm{~mL}$. Proper opening and closing behavior was assessed by analyzing slow-motion recordings (G15 PowerShot, Canon, USA). During closure, the maximum differential loading was targeted at $28 \mathrm{mmHg}$ and was automatically maintained. After each $3 \times 10^{6}$ cycles, the DTEHVs were tested again for their hydrodynamic functionality by using the hydrodynamic pulsatile test system as described above.

\section{Qualitative Tissue Analysis and Global Collagen Orientation}

\section{Histology}

Middle sections of the valves $(n=5)$ were fixed overnight in $3.7 \%$ formalin (Fluka, USA) at $4{ }^{\circ} \mathrm{C}$. After washing in PBS, the samples were embedded in TissueTek (Sakura, the Netherlands) and cured gradually in liquid nitrogen vapor. Cryosections were cut at $10 \mu \mathrm{m}$ thickness and stained for Hematoxylin and Eosin (H\&E) to assess general tissue formation and the effectiveness of the decellularization procedure, Masson Trichrome (MTC) (Staining kit, Sigma, USA) for the presence and distribution of collagen, and Elastica van Gieson (EvG) (Staining kit, Merck, Germany) for the presence of elastic matrix formation. The samples were embedded in Entellan (Merck, Germany) and analyzed using bright field microscopy (Axio Observer Z1, Zeiss, Germany) in the mid regions of the heart valve leaflets.

\section{Confocal Microscopy}

Half leaflet sections of all $(n=5)$ valves were analyzed for the effect of the insert on the global collagen orientation. Samples were stained using a whole mount staining with the collagen specific dye CNA35-OG488, ${ }^{14}$ for $1 \mathrm{~h}$ in PBS and visualized with a confocal microscope (TCS SP5X, Leica, Germany). The dye has an excitation and emission profile of 488 and 500-550 nm, respectively. Samples immerged in Mowiol (Sigma, USA) were mounted between two preparation glasses. The specimen was observed with a $\times 10$ objective and Z-stacks were made throughout $200 \mu \mathrm{m}$ of the entire arterial side of the sample using stitched adjacent tile scans. Afterwards, a maximum intensity projection was made in $Z$-direction.

\section{Tissue Mechanics and In Vivo Collagen Remodeling Simulations}

\section{Biomechanical Analyzes}

Mechanical properties of the control valve were analyzed by using a biaxial tensile tester (BioTester, 
$5 \mathrm{~N}$ load cell; CellScale, Waterloo, Canada) in combination with LabJoy software (V8.01, CellScale). Two square samples $\left(36 \mathrm{~mm}^{2}\right.$ each) per valve were symmetrically cut from the belly region. Sample thickness was measured at 3 random locations using an electronic caliper (CD-15CPX, Mitutoyo, Japan) and averaged. The samples were stretched equibiaxially in both the radial and circumferential direction up to $20 \%$ strain, at a strain rate of $100 \%$ per minute. After stretching, the samples recovered to $0 \%$ strain at a strain rate of $100 \%$ per minute, followed by a rest cycle of $54 \mathrm{~s}$. Prior to measuring the final stresses, samples were preconditioned with 5 of these cycles. A highorder polynomial curve was fitted through each individual data set in both the radial and circumferential direction. The stiffness of the tissue was represented by the tangent modulus and was defined as the tangent to the fitted polynomial curve at $20 \%$ strain.

\section{Computational Simulations}

Based on the obtained experimental mechanical data of these improved DTEHVs, computational simulations (Abaqus 6.10 Simulia, USA) as described by Loerakker et al. ${ }^{15}$ were executed to examine if the implemented changes in valve geometry resulted in reduced radial compression under pulmonary pressure conditions. The original valve design was based on the mathematical description of Thubrikar ${ }^{27}$ with the parameters $\quad R_{\mathrm{b}}=R_{\mathrm{c}}=13.5 \mathrm{~mm}, \quad H=19.15 \mathrm{~mm}$, $H_{\mathrm{s}}=3.15 \mathrm{~mm}$ and $\beta=0^{\circ}$ without any coaptation. The improved valve geometry was described by Hamid et $a l .{ }^{11}$ with the parameters $a=b=3.1 \mathrm{~mm}$, $H=18 \mathrm{~mm}, R=13.5 \mathrm{~mm}$, with $5 \mathrm{~mm}$ coaptation. All values were obtained as described in the original paper $^{15}$ and both designs used the experimentally measured tissue thickness of $0.58 \mathrm{~mm}$.

The total stress in the tissue was given by the stress in the collagen fibers (with volume fraction $\phi_{\mathrm{f}}$ ) and the isotropic matrix components (with volume fraction $\left.\left(1-\phi_{\mathrm{f}}\right)\right)^{15}$ :

$$
\boldsymbol{\sigma}=\boldsymbol{\sigma}_{\mathrm{m}}+\boldsymbol{\sigma}_{\mathrm{f}}
$$

The isotropic matrix stress is equal to:

$$
\boldsymbol{\sigma}_{\mathrm{m}}=\left(1-\phi_{\mathrm{f}}\right)\left(\kappa \frac{\ln J}{J} \mathbf{I}+\frac{G}{J}\left(\mathbf{B}-J^{\frac{2}{3}} \mathbf{I}\right)\right.
$$

with $\mathbf{B}=\mathbf{F} \cdot \mathbf{F}^{\mathrm{T}}$ the left Cauchy-Green tensor, $\mathbf{F}$ the deformation gradient tensor, $J=\operatorname{det}(\mathbf{F})$ the volumetric change ratio. The tissue was modeled as almost incompressible $(v=0.498)$, and the shear modulus $G$ was set to $10 \mathrm{kPa}$ to prevent numerical instabilities. The compression modulus is defined as:

$$
\kappa=\frac{2 G(1+v)}{3(1-2 v)} .
$$

The collagen fibers were modeled with an angular distribution of fibers (resolution of $3^{\circ}$ ), where the stress if given by:

$$
\boldsymbol{\sigma}_{\mathrm{f}}=\sum_{i=1}^{N} \varphi_{\mathrm{f}}^{i} \sigma_{\mathrm{f}}^{i} \vec{e}_{\mathrm{f}}^{i} \overrightarrow{\mathrm{e}}_{\mathrm{f}}^{i}
$$

with $\mathrm{N}$ the number of fiber directions, $\varphi_{\mathrm{f}}^{i}$ the collagen volume fraction in direction $i\left(\sum_{i=1}^{N} \varphi_{\mathrm{f}}^{i}=\phi_{\mathrm{f}}\right), \sigma_{\mathrm{f}}^{i}$ the magnitude of the stress in each fiber direction, and $\overrightarrow{\mathrm{e}}_{\mathrm{f}}^{i} \mathrm{a}$ unit vector in the deformed fiber direction $i$. The magnitude of the stress is a function of the fiber stretch $\lambda_{\mathrm{f}}$ :

$$
\sigma_{\mathrm{f}}= \begin{cases}k_{1} \lambda_{\mathrm{f}}^{2}\left(\mathrm{e}^{k_{2}\left(\lambda_{\mathrm{f}}^{2}-1\right)}-1\right) & \text { for } \lambda_{\mathrm{f}} \geq 1 \\ 0 & \text { for } \quad \lambda_{\mathrm{f}}<1\end{cases}
$$

The collagen volume fractions are described with a Gaussian distribution:

$$
\varphi_{\mathrm{f}}^{i}=\mathrm{A} \exp \left(\frac{-\left(\gamma^{i}-\mu\right)^{2}}{2 \sigma^{2}}\right)
$$

with $\gamma^{i}$ the angle of fiber direction $i$ with respect to the circumferential direction, $\mu$ the main fiber angle $\left(0^{\circ}\right), \sigma$ the standard deviation, and A a scaling factor to ensure that $\sum_{i=1}^{N} \varphi_{\mathrm{f}}^{i}=\varphi_{\mathrm{f}}$. Parameters $k_{1}, k_{2}$, and $\sigma$ were fitted to the equibiaxial tensile test data of the control valve according to the least squares method. The effect of collagen anisotropy on tissue loading was investigated by comparing the experimentally determined collagen anisotropy to complete collagen isotropy. Eventually, the computational model simulated the radial and circumferential strains in the DTEHV leaflets, by applying a mean pulmonary differential pressure of $15 \mathrm{mmHg}$ over the valve, using both the original as well as the improved geometry.

\section{Statistics}

To analyze DTEHV fatigue behavior over time, a linear regression analysis was performed (Prism V6.0d, Graphpad, USA) on the closing volume, leakage volume, cardiac output, and the effective orifice area, with a slope being significantly non-zero for $p<0.05$. Time points were averaged, and represented by their mean \pm standard deviation.

For the biomechanical analysis, samples were averaged per valve, and represented by their mean \pm standard deviation. The presence of anisotropy was defined as a significant difference $(p<0.05)$ between the obtained stiffnesses in both radial and

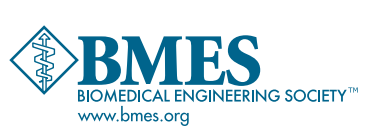


circumferential direction, analyzed using a paired student $t$ test (Prism V6.0d, Graphpad, USA).

\section{RESULTS}

Production and Functionality of the Bioreactor Insert

\section{Production of the Insert}

The improved heart valve geometry as suggested by the computational simulations, was successfully translated into a physical rigid object (Fig. 1a), matching the exact same geometry. The surface of the insert was smooth and the holes were equally distributed over the entire surface to enable sufficient nutrient exchange to the scaffold. There was sufficient space between the individual posts to prevent leaflet fusion during culture. The insert fitted nicely into the DPD bioreactor system without obstructing the pulsating flow passing by the ventricular side towards the arterial side (Fig. 1b).

\section{Functioning of the Insert}

During the 4 weeks of culturing, the leaflets of the TEHVs compacted tightly around the insert, adapting to the imposed geometry. After decellularization, the

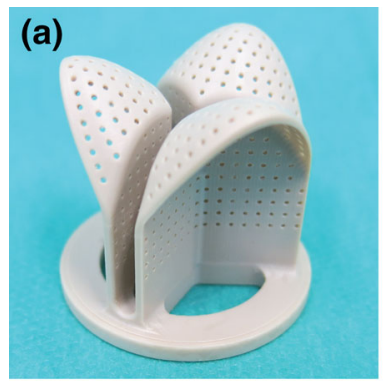

(b)
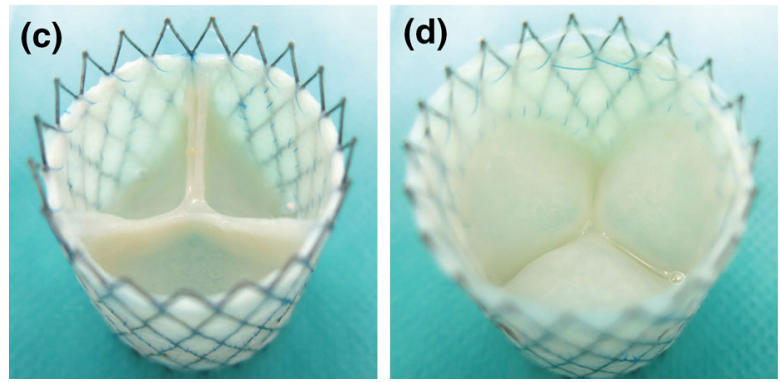

FIGURE 1. Bioreactor insert and representative DTEHV results. The developed bioreactor insert, used to impose and control the heart valve geometry during culture (a). A schematic representation of the positioning of the insert depicted in green, inside the bioreactor setup with the heart valve indicated in red, the nitinol stent in black and the pulsatile medium flow as a blue arrow (b). Two representative pictures of a human cell based DTEHVs cultivated by using the insert, from a top (c) and bottom view (d), showing large coaptation areas and an profound belly curvature, matching the shape of the bioreactor insert. valves maintained their shape, and there was no leaflet retraction visible upon removal of the insert. The coaptation area was approximately $5 \mathrm{~mm}$ in length (Fig. 1c), and the belly region of the DTEHVs maintained the imposed curvature (Fig. 1d). Tissue was uniformly distributed throughout the leaflets, without any damage or other macroscopically detectable irregularities.

\section{Hemodynamic Functionality and Fatigue Resistance}

DTEHVs $(n=4)$ were subjected to physiological pulmonary conditions in a hydrodynamic test setup. A representative graph of one valve is shown in Fig. 2. Overall, all valves opened up completely (Figs. 2b-2d), and closed symmetrically (Figs. 2e-2f). Prolapse was not observed in any of the valves.

For the long-term durability assay, valve opening and closing behavior was set to be comparable to the physiological behavior as observed in the hydrodynamic setup. From the DTEHVs $(n=4)$ subjected to fatigue tests, three remained functional up to about 12 million cycles, representative for 16 weeks in vivo follow-up time. One valve failed at 4 million cycles, and was unable to maintain stable pressure conditions during fatigue testing from the start. The valves showed no decrease in functionality over time by having an initial regurgitation fraction of $4.13 \pm 1.44 \%$, consisting out of a closing volume percentage of $3.41 \pm 1.42 \%$, and a leakage volume

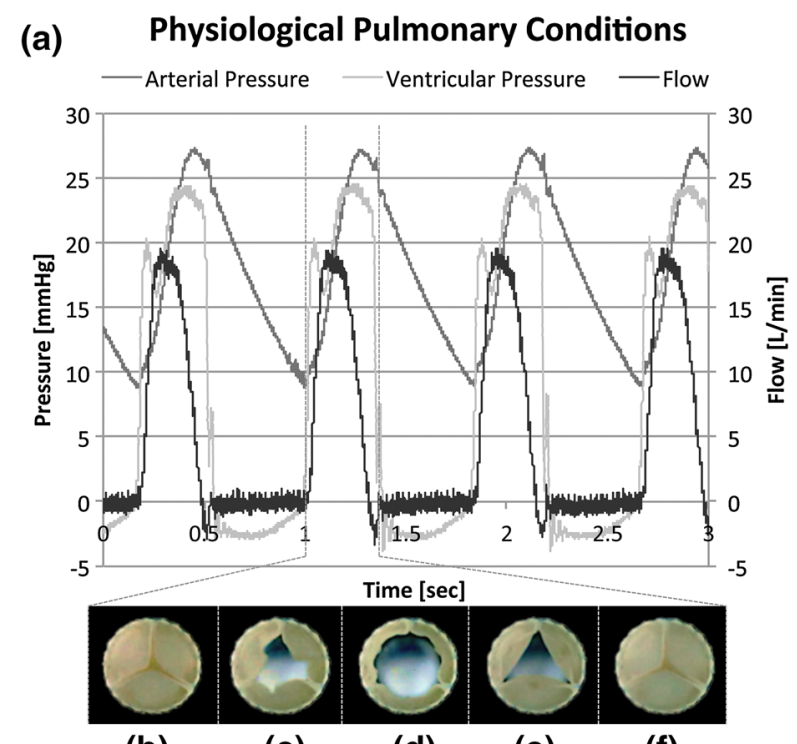

(b)

(c)

(d)

(e)

(f)

FIGURE 2. Physiological hydrodynamic pulmonary in vitro test results. Representative results of a DTEHV subjected to in vitro hydrodynamic physiological pulmonary pulsatile stimulation after 12 million cycles, showed competent valve functioning as indicated in the graph (a). Valves were opening completely (b-d) and closed symmetrically (e, f). 
percentage of only $0.73 \pm 0.08 \%$ (Fig. 3a). The valves maintained a consistent effective orifice area of $2.37 \pm 0.04 \mathrm{~cm}^{2}$, with a maintained cardiac output of $4.80 \pm 0.11 \mathrm{~L} / \mathrm{min}$ over time (Fig. $3 \mathrm{~b}$ ). Loss in functionality was sudden and in all cases the result of leaflet rupture at one of the commissure points.

\section{Qualitative Tissue Analysis and Global Collagen Orientation}

\section{Histological Appearance}

Histology was performed on samples of all DTEHVs. Representative photographs in Fig. 4, show that the tissue was uniformly distributed throughout the thickness of the leaflets. Furthermore, no necrotic or damaged tissue was observed. The decellularization procedure successfully removed all cells as demonstrated by the H\&E staining, although scaffold remnants were still present in the tissues (Fig. 4a). From the MTC staining it appeared that the leaflets are mainly composed of collagen (Fig. 4b), with no signs of elastic matrix formation in the $\mathrm{VvG}$ staining (Fig. 4c), as would otherwise be indicated by black fibers.

\section{Global Collagen Orientation}

By analyzing the collagen whole mount stainings, global collagen orientation was visualized throughout $\sim 200 \mu \mathrm{m}$ in depth, over the entire half of 5 DTEHV leaflets. A representative maximum projection in $Z$-direction is shown in Fig. 5a. Here, the collagen was clearly aligned in circumferential direction in the coaptation area (Fig. 5b) and more randomly distributed towards the bottom region of the belly (Fig. 5c).

\section{Tissue Mechanics and In Vivo Collagen Remodeling Simulations}

\section{Biomechanical Properties}

The averaged tensile curves from the control valve showed Cauchy stresses ranging between $200-300 \mathrm{kPa}$ in radial direction and $300-400 \mathrm{kPa}$ in circumferential direction at $20 \%$ strain (Fig. 6a), indicative for tissue anisotropy. From the averaged equibiaxial tensile tests on all valves, it appeared that the tangent moduli were significantly higher in circumferential direction, compared to the radial direction $(p=0.035)$, being $3.59 \pm 0.95$ and $2.47 \pm 0.74 \mathrm{MPa}$ respectively at $20 \%$ strain (Fig. 6b).

\section{Computational Simulations}

Fitting the numerical model on the experimental tensile data of the control group (Fig. 6b), resulted in the following parameter values: $k_{1}=22.0 \mathrm{kPa}, k_{2}=$ 7.50, and $\sigma=67.5^{\circ}$.

At a mean pulmonary differential pressure of $15 \mathrm{mmHg}$ the computational simulations revealed that the original design was subjected to leaflet tissue compression in the radial direction throughout the entire leaflet during loading (Fig. 7a). The improved valve design of these DTEHVs showed a considerable decrease in radial tissue compression as compared to the original design (Fig. 7b). Strains in the circumferential direction were maintained and comparable in both designs. Besides, the effect of the present collagen anisotropy on tissue loading in the improved design, revealed no changes in radial tissue compression compared to fully isotropic collagen orientation (Fig. 7c).
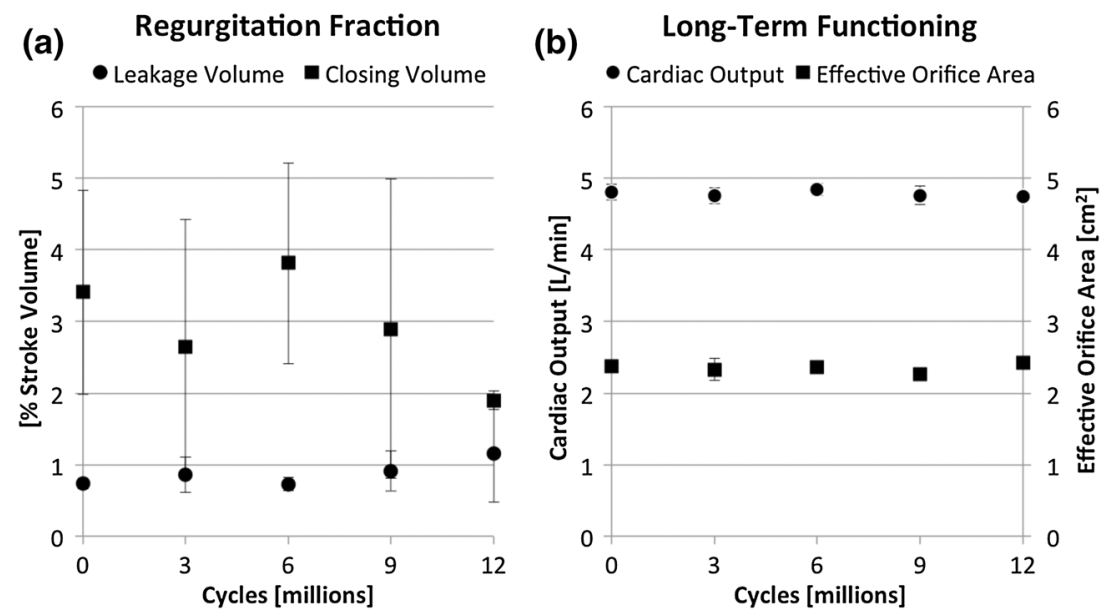

FIGURE 3. Long-term functional behavior of DTEHVs in vitro. Interval measurements of the regurgitation fraction, cardiac output, and effective orifice area, from start $(n=4)$, after 3 million cycles $(n=4), 6$ million cycles $(n=3), 9$ million cycles $(n=2)$, and after 12 million cycles $(n=2)$, showing no significant increase in regurgitation over time $(p<0.05)(a)$. Also the cardiac output and effective orifice area maintained stable over time (b). Values are represented as mean values \pm standard deviation. 

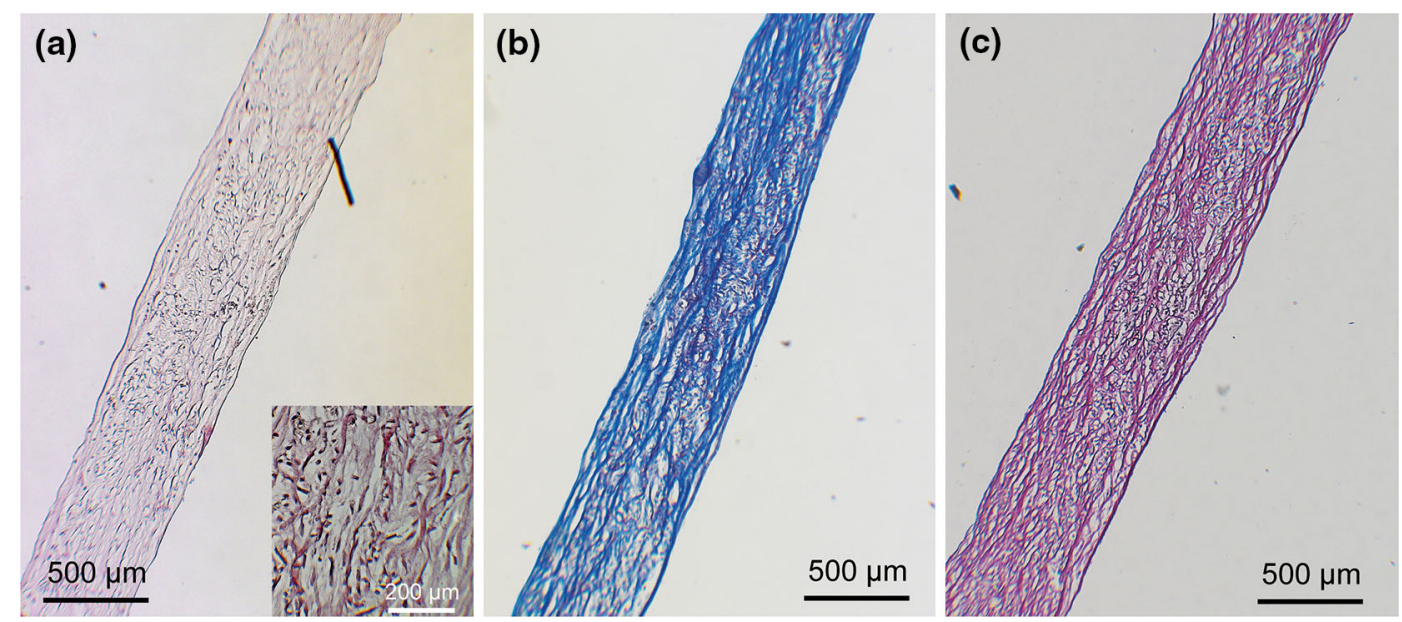

FIGURE 4. Histology on DTEHVs. Representative images of stained sections of DTEHV leaflets with Hematoxylin and Eosin, showing appropriate decellularization, equal tissue formation and the presence of scaffold remnants (a). Masson Trichrome revealed mainly collagen deposition depicted in blue (b), where Elastica van Gieson indicated no elastic matrix formation within the construct (c).

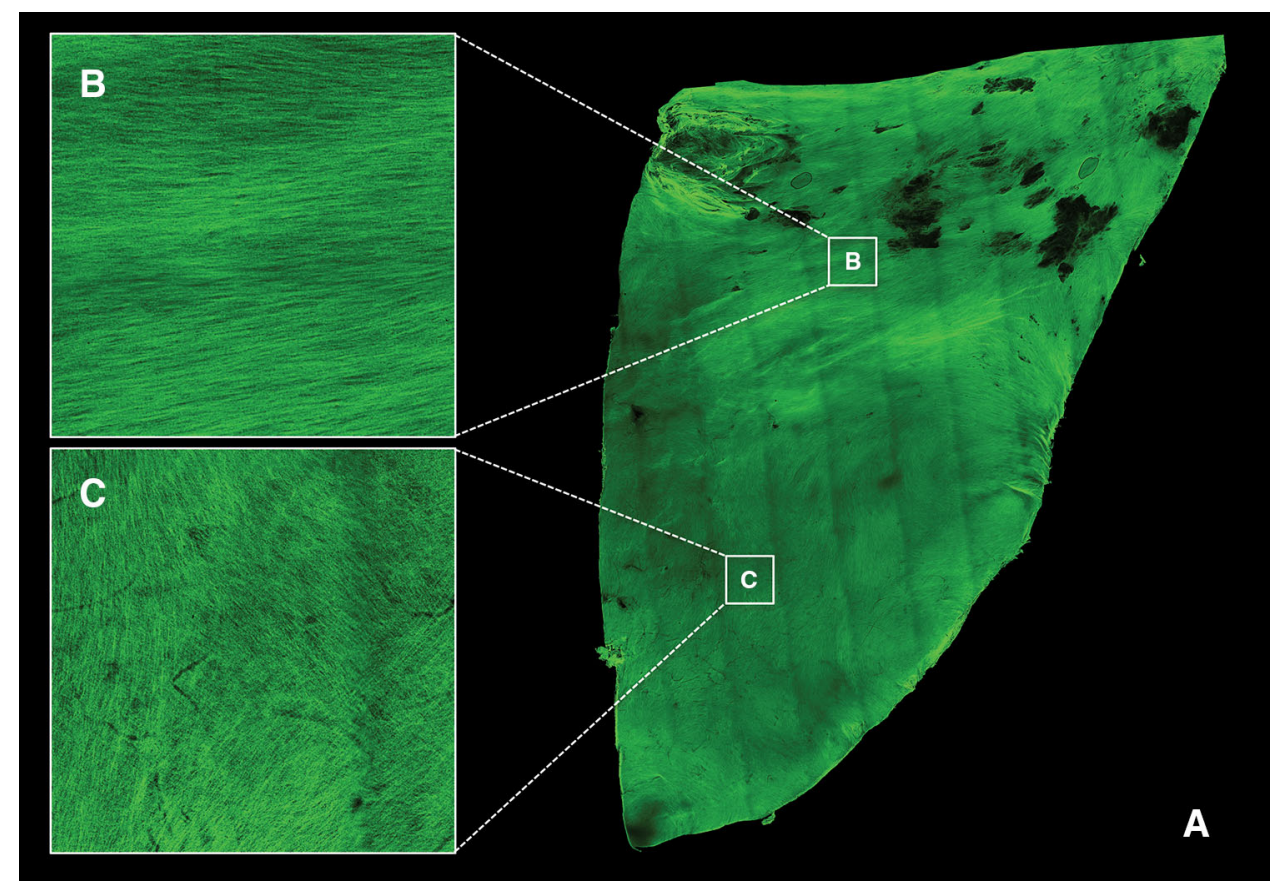

FIGURE 5. Global collagen orientation. Representative image of a collagen specific whole mount staining on half a DTEHV leaflet, visualized by confocal microscopy at the ventricular side (a), revealed that collagen orientation was clearly aligned in circumferential direction in the coaptation area (b), and randomly distributed towards the bottom of the belly (c). Black spots indicate local regions that were out of focal plane.

\section{DISCUSSION}

From our first long-term in vivo experiments where we implanted DTEHVs in sheep, we observed leaflet fusion with the wall, which resulted in the development of valvular insufficiency over time. ${ }^{6,30}$ Based on computational simulations it was hypothesized that the original DTEHV geometry had to be adjusted to enable leaflet extension in the radial direction, rather than compression. Therefore, a bioreactor insert was developed to impose the desired valvular geometry during culture, which was maintained after decellularization and removal of the insert. This resulted in DTEHVs with an increased coaptation area and a significant radial and circumferential belly curvature.

Adjusting the culture conditions in the bioreactor system by introducing a constraining insert might have affected tissue formation by limiting nutrient and 

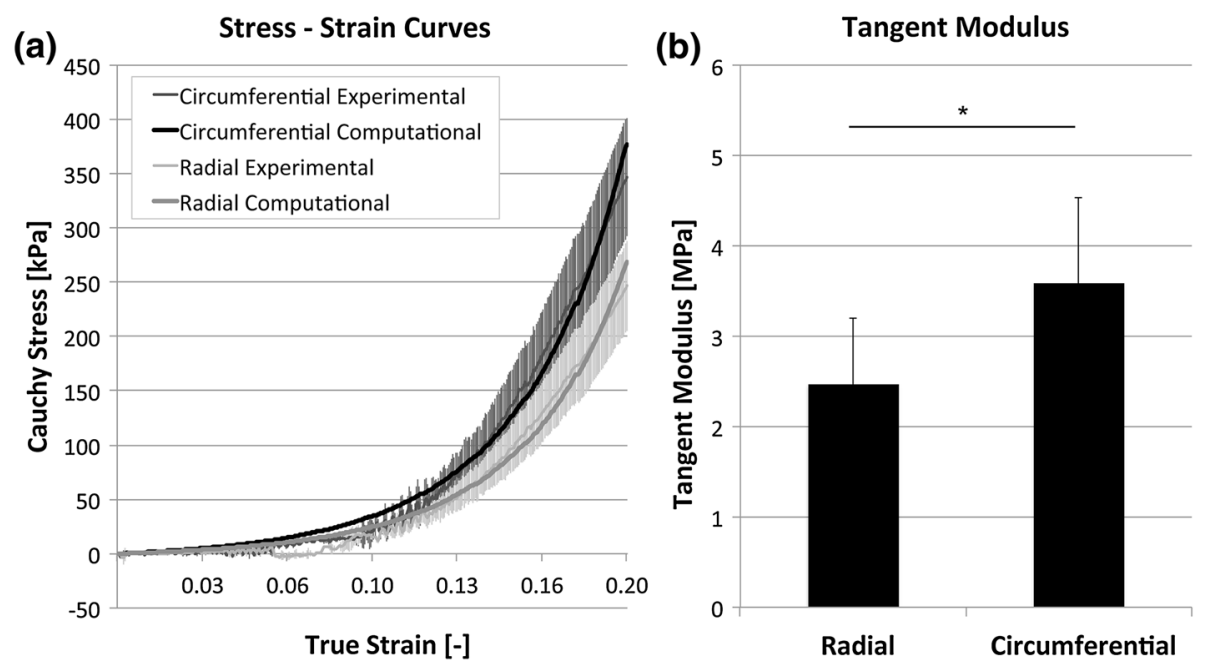

FIGURE 6. Mechanical properties of the DTEHVs. The averaged stress-strain curves obtained from equal biaxial tensile tests of the control group, in both radial and circumferential direction, shows Cauchy stresses ranging between the 200-400 kPa (a). The fitted curves are used as material parameters for the computational simulations (a). The calculated tangent moduli of all DTEHVs showed a significant increase in tissue stiffness in circumferential direction compared to the radial direction ( $\left.{ }^{\star} p=0.035\right)(b)$. All values are represented as mean values \pm standard deviation.

oxygen exchange. However, it appeared that these DTEHVs still contain a uniform ECM distribution throughout the entire thickness of the leaflets, consisting mainly of collagen. These histological findings are in agreement with previously cultured human cellbased TEHVs having the original valve geometry without the insert. ${ }^{16}$

Further more the global collagen orientation was influenced by introducing the insert. During tissue culture, non-woven PGA meshes are known to hydrolyze, thereby losing their mechanical integrity and structural support. ${ }^{9,19,23}$ This degradation profile in combination with the tension forces exerted by the cells, led to tissue compaction in the unconstrained directions, which resulted in collagen orientation along the constrained direction. ${ }^{4,18}$

In this study, tissue compaction against a rigid object was used to impose the DTEHV geometry according to the shape of the bioreactor insert. After 2 weeks of culture, the scaffold loses its mechanical integrity and the tissue starts to compact. ${ }^{29}$ While compacting, the leaflet tissue is being constrained by the rigid bioreactor insert, except for the tissue at the free edges of the leaflets that could still compact slightly in the radial direction. This resulted in mainly circumferential aligned collagen in the coaptation area, and a more random distribution towards the bottom of the belly.

Other studies in which constraining methods were used to control the geometry of DTEHVs are promising, however no long-term functionality up to 12 million cycles have been reported so far. ${ }^{18,24-26,28}$ The DTEHVs created in the present study showed satisfactory long-term functionality up to 16 weeks in vivo simulation in terms of regurgitation, cardiac output and opening and closing behavior, with only one valve failing after 4 million cycles, being unable to adapt and stabilize to the applied pulmonary pressure conditions during fatigue testing. From previous in vivo implantation studies in both sheep and nonhuman primates, host cell repopulation was observed within $5 \mathrm{~h}$, accompanied by changes in the extracellular matrix after 8 weeks, with evidence of ECM production by these cells. ${ }^{6,30}$ Therefore, the DTEHVs as developed in this study are expected to be sufficiently fatigue resistant under physiological pulmonary conditions, to provide sufficient time for host cells to repopulate and maintain the ECM.

In addition to the improved valvular geometry in terms of a large coaptation area and an enhanced belly curvature, collagen anisotropy is essential to obtain radial leaflet stretch during dynamic loading, characteristic for native leaflets, ${ }^{2}$ were anisotropy is expected to further increase after in vivo implantation because of strain-induced collagen reorganization by the repopulating host cells. ${ }^{4}$ Compared to reported stiffness values, ${ }^{1}$ human native aortic heart valve leaflets have a tangent modulus in radial and circumferential direction of about $2.0 \pm 1.5$ and $15.6 \pm 6.4 \mathrm{MPa}$ respectively. The reported tangent modulus of the DTEHVs in radial direction is comparable with $2.5 \pm 0.7 \mathrm{MPa}$, but is lower in circumferential direction with $3.6 \pm$ $1.0 \mathrm{MPa}$.

Despite that local observed differences in collagen anisotropy were not implemented into the model, these computational simulations revealed that the additional 


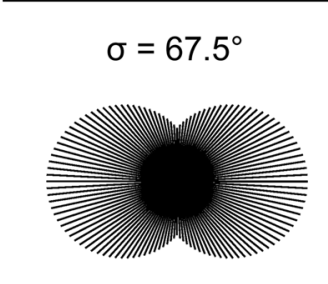

Collagen Distribution

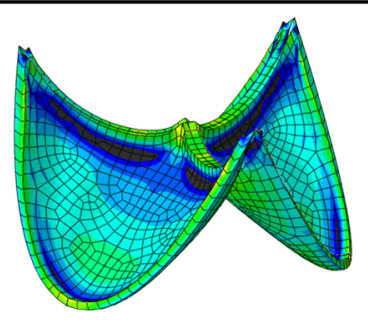

Radial

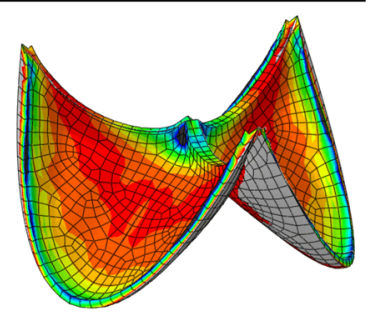

Circumferential

(b) Improved Valve Design with Anisotropic Tissue

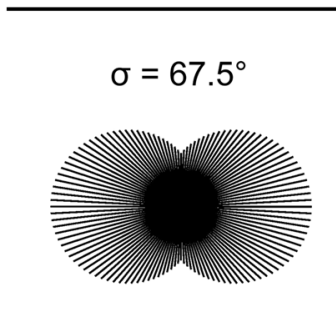

Collagen Distribution

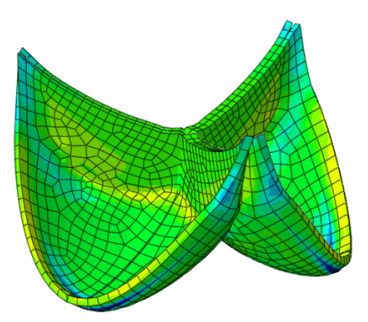

Radial

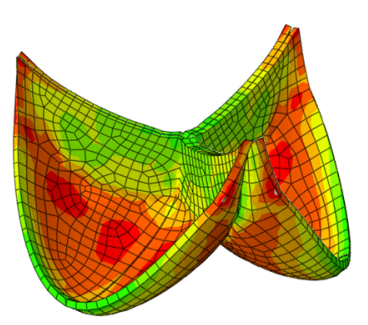

Circumferential

(c)

Improved Valve Design with Isotropic Tissue

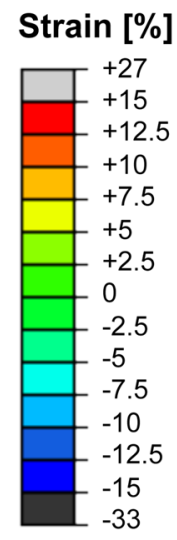

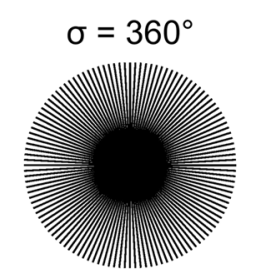

Collagen Distribution

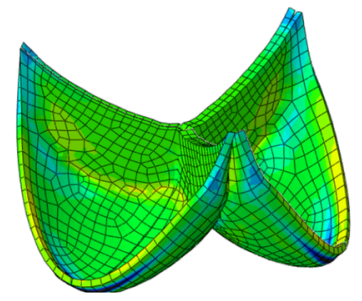

Radial

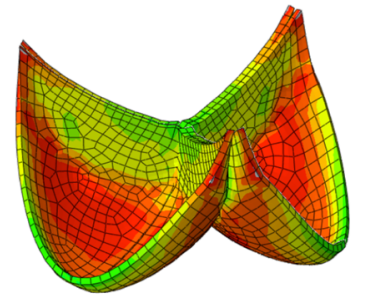

Circumferential

FIGURE 7. Computational simulations. Material parameters of the DTEHVs were implemented into an established computational valve model, to assess the strains in radial and circumferential direction in both the original and the improved valve design, simulating $15 \mathrm{mmHg}$ mean pulmonary pressure. In the original valve design the entire leaflet tissue is being compressed in radial direction (a). The improved valve design showed a considerable decrease in radial compression in the belly (b), which is primarily due to geometrical improvements rather than collagen anisotropy (c). Strains in circumferential direction are comparable between both valve designs.

effect of the overall collagen anisotropy seems not to influence the tissue loading behavior under pulmonary loading conditions. Therefore the implemented geometrical improvements only, were already sufficient to prevent radial tissue compression almost completely in the entire valve.

The concept of using DTEHVs for human applications still holds great promise in terms of regenerative capacity and growth potential, which would overcome the necessity for multiple re-interventions in young patients. The use of autologous cells is not required and allogeneic cells can be used that simplifies regulations and allows for off-the-shelf availability. Further development on biodegradable stents that are suitable for minimal invasive heart valve implantation should be focus of future studies to complete the growing valve concept.

In conclusion, this study proposes a successful solution to impose a desired three-dimensional curved tissue engineered valvular geometry by using a constraining bioreactor insert during culture, which allows for a maintained shape after decellularization and removal of the insert. This resulted in fully competent off-the-shelf available human cell-based DTEHVs with a large coaptation area and profound belly curvature. Long-term functionality was maintained mainly up to 
16 weeks in vivo simulation, allowing sufficient time for host cell repopulation. Usage of the bioreactor inserts resulted in homogeneously distributed tissue formation, circumferential collagen orientation in the coaptation region, and overall leaflet tissue anisotropy. Based on the mechanical data, our computational models revealed a considerable decrease in radial tissue compression with the obtained geometrical adjustments. Therefore, these improved DTEHV are expected to be less prone to host cell mediated leaflet retraction and will remain competent after implantation.

\section{ACKNOWLEDGMENT}

This work was financially supported by the European Union's Seventh Framework Program (FP7/ 2007-2013) under Grant Agreement Number 242008 (LifeValve).

\section{OPEN ACCESS}

This article is distributed under the terms of the Creative Commons Attribution 4.0 International License (http://creativecommons.org/licenses/by/4.0/), which permits unrestricted use, distribution, and reproduction in any medium, provided you give appropriate credit to the original author(s) and the source, provide a link to the Creative Commons license, and indicate if changes were made.

\section{REFERENCES}

${ }^{1}$ Balguid, A., M. P. Rubbens, A. Mol, R. A. Bank, A. J. J. C. Bogers, J. P. van Kats, B. A. J. M. de Mol, F. P. T. Baaijens, and C. V. C. Bouten. The role of collagen crosslinks in biomechanical behavior of human aortic heart valve leaflets-relevance for tissue engineering. Tissue Eng. 13:1501-1511, 2007.

${ }^{2}$ Billiar, K. L., and M. S. Sacks. Biaxial mechanical properties of the native and glutaraldehyde treated aortic valve cusp: Part I-experimental results. J. Biomech. Eng. 122:327-335, 2000

${ }^{3}$ Cebotari, S., I. Tudorache, A. Ciubotaru, D. Boethig, S. Sarikouch, A. Goerler, A. Lichtenberg, E. Cheptanaru, S. Barnaciuc, A. Cazacu, O. Maliga, O. Repin, L. Maniuc, T. Breymann, and A. Haverich. Use of fresh decellularized allografts for pulmonary valve replacement may reduce the reoperation rate in children and young adults: early report. Circulation 124:S115-S123, 2011.

${ }^{4}$ de Jonge, N., F. M. W. Kanters, F. P. T. Baaijens, and C. V. C. Bouten. Strain-induced collagen organization at the micro-level in fibrin-based engineered tissue constructs. Ann. Biomed. Eng. 41:763-774, 2012.
${ }^{5}$ Dijkman, P. E., A. Driessen-Mol, L. Frese, S. P. Hoerstrup, and F. P. T. Baaijens. Decellularized homologous tissue-engineered heart valves as off-the-shelf alternatives to xeno- and homografts. Biomaterials 33:4545-4554, 2012. ${ }^{6}$ Driessen-Mol, A., M. Y. Emmert, P. E. Dijkman, L. Frese, B. Sanders, B. Weber, N. Cesarovic, M. Sidler, J. Leenders, R. Jenni, J. Grünenfelder, V. Falk, F. P. T. Baaijens, and S. P. Hoerstrup. Transcatheter implantation of homologous "off-the-shelf" tissue-engineered heart valves with self-repair capacity. J. Am. Coll. Cardiol. 63:1320-1329, 2014.

${ }^{7}$ Erdbrügger, W., W. Konertz, P. M. Dohmen, S. Posner, H. Ellerbrok, O.-E. Brodde, H. Robenek, D. Modersohn, A. Pruss, S. Holinski, M. Stein-Konertz, and G. Pauli. Decellularized xenogenic heart valves reveal remodeling and growth potential in vivo. Tissue Eng. 12:2059-2068, 2006.

${ }^{8}$ Flanagan, T. C., J. S. Sachweh, J. Frese, H. Schnöring, N. Gronloh, S. Koch, R. H. Tolba, T. Schmitz-Rode, and S. Jockenhoevel. In vivo remodeling and structural characterization of fibrin-based tissue-engineered heart valves in the adult sheep model. Tissue Eng. Part A 15:2965-2976, 2009

${ }^{9}$ Generali, M., P. E. Dijkman, and S. P. Hoerstrup. Bioresorbable scaffolds for cardiovascular tissue engineering. EMJ Int. Cardiol. 1:91-99, 2014.

${ }^{10}$ Gottlieb, D., T. Kunal, S. Emani, E. Aikawa, D. W. Brown, A. J. Powell, A. Nedder, G. C. Engelmayr, J. M. Melero-Martin, M. S. Sacks, and J. E. Mayer. In vivo monitoring of function of autologous engineered pulmonary valve. J. Thorac. Cardiovasc. Surg. 139:723-731, 2010.

${ }^{11}$ Hamid, M. S., H. N. Sabbah, and P. D. Stein. Influence of stent height upon stresses on the cusps of closed bioprosthetic valves. J. Biomech. 19:759-769, 1986.

${ }^{12}$ Hoerstrup, S. P., R. Sodian, S. Daebritz, J. Wang, E. A. Bacha, D. P. Martin, A. M. Moran, K. J. Guleserian, J. S. Sperling, S. Kaushal, J. P. Vacanti, F. J. Schoen, and J. E. Mayer. Functional living trileaflet heart valves grown in vitro. Circulation 102:44-49, 2000.

${ }^{13}$ Honge, J. L., J. Funder, E. Hansen, P. M. Dohmen, W. Konertz, and J. M. Hasenkam. Recellularization of aortic valves in pigs. Eur. J. Cardiothorac. Surg. 39:829-834, 2011.

${ }^{14}$ Krahn, K. N., C. V. C. Bouten, S. van Tuijl, M. A. M. J. van Zandvoort, and M. Merkx. Fluorescently labeled collagen binding proteins allow specific visualization of collagen in tissues and live cell culture. Anal. Biochem. 350:177-185, 2006.

${ }^{15}$ Loerakker, S., G. Argento, C. W. J. Oomens, and F. P. T. Baaijens. Effects of valve geometry and tissue anisotropy on the radial stretch and coaptation area of tissue-engineered heart valves. J. Biomech. 46:1792-1800, 2013.

${ }^{16}$ Mol, A., A. I. Smits, C. V. Bouten, and F. P. Baaijens. Tissue engineering of heart valves: advances and current challenges. Expert Rev. Med. Dev. 6:259-275, 2009.

${ }^{17}$ Mol, A., N. J. B. Driessen, M. C. M. Rutten, S. P. Hoerstrup, C. V. C. Bouten, and F. P. T. Baaijens. Tissue engineering of human heart valve leaflets: a novel bioreactor for a strain-based conditioning approach. Ann. Biomed. Eng. 33:1778-1788, 2005.

${ }^{18}$ Neidert, M. R., and R. T. Tranquillo. Tissue-engineered valves with commissural alignment. Tissue Eng. 12:891903, 2006.

${ }^{19} \mathrm{Oh}, \mathrm{S} . \mathrm{H}$., and J. H. Lee. Hydrophilization of synthetic biodegradable polymer scaffolds for improved cell/tissue compatibility. Biomed. Mater. 8:1-16, 2013. 
${ }^{20}$ Pibarot, P., and J. G. Dumesnil. Prosthetic heart valves: selection of the optimal prosthesis and long-term management. Circulation 119:1034-1048, 2009.

${ }^{21}$ Rosengart, T. K., T. Feldman, M. A. Borger, T. A. Vassiliades, Jr., A. M. Gillinov, K. J. Hoercher, A. Vahanian, R. O. Bonow, and W. O'Neill. Percutaneous and minimally invasive valve procedures. Circulation 117:1750-1767, 2008.

${ }^{22}$ Schoen, F. J. Heart valve tissue engineering: quo vadis? Curr. Opin. Biotech. 22:698-705, 2011.

${ }^{23}$ Shum, A. W. T., and A. F. T. Mak. Morphological and biomechanical characterization of poly(glycolic acid) scaffolds after in vitro degradation. Polym. Degrad. Stab. 81:141-149, 2003.

${ }^{24}$ Syedain, Z. H., A. R. Bradee, S. Kren, D. A. Taylor, and R. T. Tranquillo. Decellularized tissue-engineered heart valve leaflets with recellularization potential. Tissue Eng. Part A 19:759-769, 2012.

${ }^{25}$ Syedain, Z. H., L. A. Meier, J. M. Reimer, and R. T. Tranquillo. Tubular heart valves from decellularized engineered tissue. Ann. Biomed. Eng. 41:2645-2654, 2013.

${ }^{26}$ Syedain, Z. H., M. T. Lahti, S. L. Johnson, P. S. Robinson, G. R. Ruth, R. W. Bianco, and R. T. Tranquillo. Implantation of a tissue-engineered heart valve from human fibroblasts exhibiting short term function in the sheep pulmonary artery. Cardiovasc. Eng. Tech. 2:101-112, 2011.

${ }^{27}$ Thubrikar, M. J. The Aortic Valve. Boca Raton: CRC Press, 1990

${ }^{28}$ Tremblay, C., J. Ruel, J. Bourget, V. Laterreur, K. Vallières, M. Y. Tondreau, D. Lacroix, L. Germain, and F. A. Auger. A new construction technique for tissue-engineered heart valves using the self-assembly method. Tissue Eng. Part C 20:905-915, 2014.

${ }^{29}$ van Vlimmeren, M. A. A., A. Driessen-Mol, C. W. J. Oomens, and F. P. T. Baaijens. An in vitro model system to quantify stress generation, compaction, and retraction in engineered heart valve tissue. Tissue Eng. Part C 17:983$991,2011$.

${ }^{30}$ Weber, B., P. E. Dijkman, J. Scherman, B. Sanders, M. Y. Emmert, J. Grünenfelder, R. Verbeek, M. Bracher, M. Black, T. Franz, J. Kortsmit, P. Modregger, S. Peter, M. Stampanoni, J. Robert, D. Kehl, M. van Doeselaar, M. Schweiger, C. E. Brokopp, T. Wälchli, V. Falk, P. Zilla, A. Driessen-Mol, F. P. T. Baaijens, and S. P. Hoerstrup. Offthe-shelf human decellularized tissue-engineered heart valves in a non-human primate model. Biomaterials 34:7269-7280, 2013. 